Quim. Nova, Vol. 34, No. 9, 1672-1677, 2011

\title{
ACTITUDES HACIA LA QUÍMICA DE ESTUDIANTES DE DIFERENTES CARRERAS UNIVERSITARIAS EN COLOMBIA
}

\author{
Manuel F. Molina, José G. Carriazo* y Diana M. Farías \\ Departamento de Química, Facultad de Ciencias, Universidad Nacional de Colombia, Carrera 30 No. 45-03, Bogotá, Colombia
}

Recebido em 15/3/11; aceito em 2/5/11; publicado na web em 17/6/11

\begin{abstract}
STUDENTS' ATTITUDES TOWARD CHEMISTRY IN DIFFERENT UNDERGRADUATE PROGRAMS IN COLOMBIA. This work reports a study on the attitudes of Colombian students towards Chemistry in different undergraduate programs. The research was performed on 769 students belonging to several Chemistry courses, by applying an adaptation of Salta and Tzougraki's test and using Likert's scale. The results revealed that Chemistry is being considered as difficult and little useful, but very interesting. Thus, the difficulty to learn Chemistry may be associated with its language and abstract conceptual nature. In addition, more attention should be given to the class contents and methodologies provided to the students, in order to engage them with those interest aspects and meaningful topics of each program.
\end{abstract}

Keywords: attitudes toward chemistry; chemical education; science teaching.

\section{INTRODUCCIÓN}

Los problemas sobre el aprendizaje de las ciencias, en particular de la química, frecuentemente se ponen en evidencia al indagar a profesores encargados de impartir esta disciplina. Expresiones como "no quieren aprender", "nada les interesa", "no saben nada" o "sólo estudian para la prueba", hacen parte comúnmente de juicios valorativos generalizados emitidos desde algunos profesores hacia sus estudiantes. Las expresiones anteriores involucran de manera global las actitudes de los estudiantes hacia las ciencias, un objeto de investigación actual en el campo de la educación, las cuales se han estudiado más de forma general que hacia las disciplinas en particular. Las actitudes hacia las ciencias están estrechamente ligadas con los logros académicos, y el desarrollo de actitudes positivas en los estudiantes hacia las disciplinas científicas constituye una de las grandes responsabilidades de cada profesor de ciencias. ${ }^{1,2}$ Además, las actitudes de los estudiantes hacia la ciencia constituyen un factor importante que ejerce influencia sobre la motivación. ${ }^{3}$ Osborne caracteriza las actitudes hacia el aprendizaje de las ciencias como algo urgente de investigar debido a razones que tienen que ver con: ${ }^{4}$ disminución de personas que quieren estudiar ciencias, disminución en el rendimiento escolar, la importancia del conocimiento científico para afrontar problemas sociales y la relación entre utilidad económica y conocimiento científico. De otro lado, la profesión docente impone como principal preocupación el aprendizaje (construcción de conceptos, actitudes y valores y la generación de competencias) valorado en forma global por los rendimientos y puesto en evidencia por los porcentajes de pérdida en los cursos. De esta forma, el presente trabajo parte de la inquietud de indagar más acerca de los factores involucrados en el bajo rendimiento académico y los elevados porcentajes de pérdida en los cursos de química general de la Universidad Nacional de Colombia, los cuales pueden oscilar entre 10-60\% (por profesor o curso), con una población de estudiantes heterogénea por estar compuesta por personas de varias carreras. Se investigó sobre las actitudes de los

\footnotetext{
*e-mail: jcarriazog@unal.edu.co
}

estudiantes hacia la química con el objeto de poseer un conocimiento que permita a futuro realizar propuestas que mejoren su desempeño en los cursos, así como modificaciones curriculares.

Las investigaciones sobre actitudes hacia las ciencias se han realizado sobre todo en la etapa de educación secundaria y media; por ejemplo, Marba-Tallada y Márquez estudian las opiniones de los alumnos hacia la ciencia escolar encontrando que las actitudes positivas decaen a lo largo de la educación secundaria por factores como el desencanto con los contenidos, ${ }^{5}$ los cuales pueden ser muy académicos y acercarse a cursos preuniversitarios, como se ha observado en química. ${ }^{6}$ En la misma dirección, Kind et al. encuentran en un estudio de 6 factores (aprendizaje de la ciencia, auto concepto de ciencia, trabajo práctico, ciencia fuera de la escuela, futura participación en ciencia e importancia de la ciencia) que todos decaen entre los grados 7-9, pero entre ellos el trabajo práctico trata de mantenerse, siendo éste el más relevante porque los estudiantes consideran que las actividades prácticas generan un mejor aprendizaje y son más importantes frente a las clases magistrales, abstractas y tediosas. ${ }^{7}$ Otros estudios importantes sobre actitudes hacia las ciencias, y en particular hacia la química, en estudiantes de secundaria fueron desarrollados por Shannon et al.,${ }^{8}$ Salta y Tzougraki, ${ }^{9}$ Barnes et al. ${ }^{10}$ y Cheung ${ }^{1}$ en diferentes países. Las investigaciones anteriores han mostrado que, las actitudes positivas hacia las ciencias básicas en general pueden cambiar de acuerdo al grado, género y contexto social en que se encuentran inmersos los estudiantes, conformando un evento más complejo de lo previsto y revelando la necesidad de abordar este tipo de estudios en niveles de educación diferentes y en regiones distintas. En efecto, Wang y Berlin ${ }^{3}$ consideraron conveniente la construcción y validación de un nuevo instrumento para medir las actitudes hacia la ciencia en estudiantes de escuelas taiwanesas, teniendo en cuenta que dichos estudiantes pertenecen a un contexto cultural diferente y poseen bases educativas distintas al mundo occidental. Finalmente, uno de los resultados más importantes de Cheung, sobre los estudiantes de Hong Kong, indica que los profesores de química requieren tomar en consideración diferentes componentes del currículo con el objeto de mejorar las actitudes hacia la química, como por ejemplo incorporar el aprendizaje basado en investigación para el desarrollo 
de actividades de laboratorio y el uso de las aproximaciones humanísticas de la ciencia. ${ }^{1}$

El estudio de las actitudes a nivel de países de habla hispana es pequeño en comparación a los anglosajones, sin embargo autores como Vázquez y Manassero poseen varias publicaciones al respecto. Recientemente Vázquez y Manassero concluyen, sobre una población de estudiantes españoles de secundaria, que la imagen de ciencia no sufre cambios importantes en la escuela, pero que en general las actitudes positivas hacia la ciencia escolar decrecen constantemente. ${ }^{11}$ Los diferentes estudios establecen la diferencia entre lo que se puede pensar de la ciencia y la actitud que se tiene frente al estudio y el aprendizaje de la ciencia en la escuela, cuestión de mayor interés para los profesores.

\section{El concepto de actitud}

En relación al concepto de actitud, éste ha sido ya debatido por autores como Ajzen, ${ }^{12}$ Osborne,${ }^{4}$ Reid ${ }^{13}$ y otros. En el presente trabajo tomamos el concepto de actitud propuesto por Kind et al.: conjunto de sentimientos que una persona tiene acerca de un objeto, basado en sus creencias acerca del objeto. ${ }^{7}$ Con esta definición las actitudes hacia la química corresponden a las opiniones cognitivas y emocionales acerca de varios aspectos de la química, los cuales derivamos del estudio de Salta y Tzougraki: ${ }^{9}$ la importancia de la química en la vida; la importancia del curso de química; la dificultad del curso de química; el interés por el curso de química y la utilidad del curso de química en el futuro de una profesión.

Salta y Tzougraki desarrollaron y aplicaron un instrumento para conocer las actitudes hacia la química en estudiantes de grado 11 en Grecia (12 es el último grado) persiguiendo conocer el efecto del género en las actitudes, y la correlación entre actitudes hacia la química y sus logros en química. ${ }^{9}$ Por ser diferentes las poblaciones, la de Salta y Tzougraki respecto a la del presente estudio, no tendremos en cuenta el género, ya que no se pretende establecer relaciones genero-actitud sino carrera-actitud. Adicionalmente, los estudiantes pertenecientes a la población del presente estudio ya han elegido su futura profesión; y por tanto, se busca saber el efecto de la especialidad (carrera) ya que todos toman un curso de química diseñado probablemente para químicos o afines, y no para una población heterogénea en profesiones. Finalmente, no estableceremos una correlación con los logros de los estudiantes. Con la intención de lograr establecer comparaciones interculturales, la adaptación del instrumento de Salta y Tzougraki comprende una primera parte del estudio y luego la aplicación corresponde a la parte central donde responderemos ampliamente la pregunta ¿qué efecto tiene la carrera sobre las actitudes de estudiantes dentro de un curso de química general?

\section{METODOLOGIA}

La investigación fue realizada con estudiantes de la Universidad Nacional de Colombia, sede Bogotá, los cuales tomaron el curso de
Química General en el segundo semestre de 2010. Se encuestaron 769 estudiantes (309 mujeres y 460 hombres) pertenecientes a 17 cursos con diferentes profesores. Los participantes poseen edades entre $16 \mathrm{y}$ 29 años con un promedio de edad de 18,6 años, relacionado con una mayoría perteneciente a primer semestre (alrededor del $90 \%$ ) y otros distribuidos entre segundo y séptimo semestre. La Tabla 1 muestra la distribución de estudiantes participantes por carrera y género.

El cuestionario fue adaptado de Salta y Tzougraki, ${ }^{9}$ siguiendo las consideraciones de Hambleton, resumidas a continuación: ${ }^{14}$ a) traducción del cuestionario teniendo en cuenta el idioma y la cultura. Esta traducción se realizó por expertos en el área y en el idioma; b) revisión de la versión adaptada del cuestionario por 5 expertos en investigación didáctica; c) realización de un estudio piloto a la versión adaptada; d) estudio de la consistencia interna del cuestionario; e) aplicación del cuestionario final, el cual no tuvo modificaciones luego de la prueba piloto. El cuestionario completo se puede ver en la Figura 1. El cuestionario consta de 23 preguntas, 13 redactadas de forma positiva y 10 de forma negativa, buscando evitar tendencias al responder. Las preguntas están distribuidas en cuatro categorías, de

\begin{tabular}{|c|c|c|c|c|c|c|}
\hline \multicolumn{7}{|c|}{ CARRERA_ } \\
\hline 1. & El curso de química me parece más agradable que otros cursos. & TA & A & 1 & D & TD \\
\hline 2. & Los símbolos utilizados en la clase de química me parecen difíciles de entender. & TA & A & 1 & D & TD \\
\hline 3. & Me gustaria tener clases de química con mayor frecuencia. & TA & A & 1 & D & TD \\
\hline 4. & La química sirve para conocer muchos aspectos de nuestra vida cotidiana. & TA & A & 1 & D & TD \\
\hline 5. & Resuelvo con facilidad los problemas de química. & TA & A & 1 & D & TD \\
\hline 6. & Me aburro durante las clases de química. & TA & A & 1 & D & TD \\
\hline 7. & El conocimiento de química solo me sirve para pasar el curso de química. & TA & A & 1 & D & TD \\
\hline 8. & El desarrollo de la química ha mejorado nuestra calidad de vida. & TA & A & 1 & D & TD \\
\hline 9. & La esperanza de resolver muchos problemas ambientales está en la química. & TA & A & 1 & D & TD \\
\hline 10. & La seguridad de mi futuro es independiente del conocimiento químico. & TA & A & 1 & D & TD \\
\hline 11. & El progreso del país está relacionado con la industrialización química. & TA & A & 1 & D & TD \\
\hline 12. & La quimica es una ciencia muy compleja para mi nivel deconocimientos. & TA & A & 1 & D & TD \\
\hline 13. & Debo esforzarme mucho para aprender quimica. & TA & A & 1 & D & TD \\
\hline 14. & El lenguaje de la química y sus símbolos son fáciles de entender. & TA & A & 1 & D & TD \\
\hline 15. & La profesión de químico es poco interesante. & TA & A & 1 & D & TD \\
\hline 16. & Todas las carreras de la universidad deberían tener al menos un curso de química. & TA & A & 1 & D & TD \\
\hline 17. & Detesto los cursos de química. & TA & A & 1 & D & TD \\
\hline 18. & Los conocimientos en química son necesarios en el desarrollo de mi carrera & TA & A & 1 & D & TD \\
\hline 19. & Desearía que el programa de mi carrera actual tuviera menos clases de química & TA & A & 1 & D & TD \\
\hline 20. & Comprendo los conceptos de química con facilidad & TA & A & 1 & D & TD \\
\hline 21. & El curso de química es muy interesante & TA & A & 1 & D & TD \\
\hline 22. & Cuando me enfrento a los problemas de químicaNO puedo resolverlos con facilidad. & TA & A & 1 & D & TD \\
\hline 23. & Consideraría cambiarme de carrera y tener una profesión relacionada con la química. & TA & A & 1 & D & TD \\
\hline
\end{tabular}

Figura 1. Cuestionario de actitudes hacia la química. Opciones de respuesta: $T A=$ totalmente de acuerdo. $A=$ de acuerdo. $I=$ no estoy seguro(a): Indecisión. $D=$ en desacuerdo. $T D=$ totalmente en desacuerdo

Tabla 1. Distribución de la población encuestada por carrera y género

\begin{tabular}{|c|c|c|c|c|c|c|c|c|c|c|c|c|c|c|c|c|c|c|}
\hline $\begin{array}{l}\text { Carrera/ } \\
\text { género }\end{array}$ & 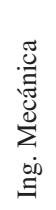 & 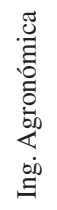 & $\begin{array}{l}\frac{\pi}{00} \\
\frac{0}{0} \\
\frac{0}{01}\end{array}$ & 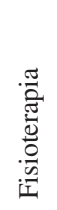 & 罵 & 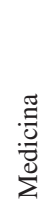 & $\begin{array}{l}\vec{Z} \\
\text { id } \\
\Xi\end{array}$ & 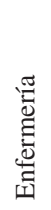 & $\begin{array}{l}\frac{\pi}{00} \\
\frac{0}{0} \\
0 \\
0\end{array}$ & 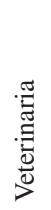 & 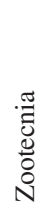 & 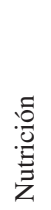 & 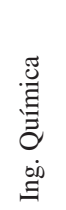 & $\begin{array}{l}\frac{\pi}{0} \\
\frac{0}{0} \\
0 \\
\stackrel{0}{0} \\
0 \\
0\end{array}$ & 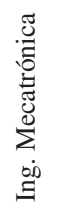 & 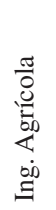 & 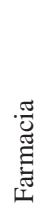 & $\frac{\tilde{o}}{\tilde{0}}$ \\
\hline Mujeres & 5 & 8 & 18 & 9 & 29 & 18 & 15 & 38 & 17 & 16 & 3 & 16 & 54 & 26 & 2 & 5 & 30 & 309 \\
\hline Hombres & 50 & 17 & 22 & 3 & 41 & 29 & 32 & 28 & 20 & 14 & 17 & 5 & 64 & 17 & 23 & 25 & 53 & 460 \\
\hline Total & 55 & 25 & 40 & 12 & 70 & 47 & 47 & 66 & 37 & 30 & 20 & 21 & 118 & 43 & 25 & 30 & 83 & 769 \\
\hline
\end{tabular}


la siguiente forma: importancia de la química y del curso de química (preguntas 4, 8, 9, 11 y 16); dificultad en el estudio y comprensión de la química (preguntas 2, 5, 13, 14, 20 y 22); interés por el curso de química (preguntas $1,3,6,7,12,15,17,19$ y 21) y utilidad del conocimiento químico (preguntas 10, 18 y 23).

Una vez culminada la adaptación del cuestionario, se realizó la aplicación de la encuesta y luego su selección teniendo en cuenta que los cuestionarios hayan sido clara y completamente diligenciados. Para facilitar que otros profesores-investigadores en diferentes lugares puedan aplicar el cuestionario y realizar el tratamiento estadístico, usamos el programa Excel asignando un valor a cada opción de la escala Likert, de 1 a 5 para las preguntas que están en forma positiva, así: $\mathrm{TA}=5, \mathrm{~A}=4, \mathrm{I}=3, \mathrm{D}=2$ y $\mathrm{TD}=1 ;{ }^{15}$ mientras que para las preguntas en forma negativa la escala se invierte, de forma similar a lo propuesto por Vázquez y Manassero (preguntas 2, 6, 7, 10, 12, 13, 15, 17, 19, 22). ${ }^{16}$ De esta forma un valor más cercano a 5 muestra siempre una actitud más positiva. Se promedian los valores según la cantidad de estudiantes, para obtener valores entre 1 y 5 . Se define el valor 3 como neutral, valores entre 2-3 representan actitudes negativas, entre 3-4 actitudes moderadamente positivas y valores entre $4-5$ representan actitudes muy positivas.

\section{RESULTADOS Y DISCUSIÓN}

\section{Prueba piloto}

La prueba piloto se aplicó a 46 estudiantes, donde no se hizo distinción de género. En la Tabla 2 se ve la cantidad de estudiantes que seleccionaron cada opción de respuesta en los diferentes ítems; colocamos la sumatoria de las respuestas al final (en la columna total) para verificar el correcto ingreso de los datos. A la derecha, como valores, aparecen los puntajes que tiene cada opción según el número de respuestas y el valor asignado; por ejemplo, 10 para el ítem 1 con opción TA (columna 8, fila 2) representa la multiplicación de $2 \times 5$, y al lado 76 proviene de multiplicar 19x4 (ítem 1, opción A); se suman todos los valores y se divide entre 46 para obtener el promedio, 3,2 para el ítem 1 (El curso de química me parece más agradable que otros cursos). En el ítem 2 (Los símbolos utilizados en la clase de química me parecen difíciles de entender) se realizó un cálculo contrario por ser un ítem enunciado de forma negativa, ya que de no ser así la escala daría valores más bajos, respecto a 5, e indicaría actitudes más negativas. El promedio obtenido de 3,5 (ítem 2) indica una actitud moderadamente positiva, o sea que a los estudiantes les parece que los símbolos utilizados en la clase de química son fáciles de entender. El resto de cálculos son similares y los realiza el programa Excel para cada ítem.

La consistencia interna de la prueba fue estimada con el alfa de Cronbach aplicando la fórmula de la varianza de los ítems, dando un valor de 0,81 , que indica que los datos son confiables y comparables con los obtenidos por Salta y Tzougraki cuyo valor resultante en la prueba piloto fue de $0,89 .{ }^{9}$ En estudios anteriores se acepta que un valor superior a 0,7 indica una consistencia adecuada de la prueba. ${ }^{1,14}$

Se determinó el alfa para cada una de las dimensiones utilizando toda la población: importancia de la química y del curso de química, 0,83; dificultad en el estudio y comprensión de la química, 0,91 ; interés por el curso de química, 0,81 y utilidad del conocimiento químico 0,80 . Salta y Tzougraki, en el mismo orden anterior obtienen valores de alfa de 0,$87 ; 0,89 ; 0,71$ y 0,67 respectivamente. ${ }^{9}$ Las categorías

Tabla 2. Resultados de la prueba piloto de actitudes hacia la química

\begin{tabular}{|c|c|c|c|c|c|c|c|c|c|c|c|c|c|}
\hline ítem & $\mathrm{TA}$ & A & I & $\mathrm{D}$ & TD & Total & & & Valores & & & ítem & Prom. \\
\hline 1 & 2 & 19 & 13 & 11 & 1 & 46 & 10 & 76 & 39 & 22 & 1 & 1 & 3,2 \\
\hline 2 & 2 & 8 & 8 & 21 & 7 & 46 & 2 & 16 & 24 & 84 & 35 & 2 & 3,5 \\
\hline 3 & 4 & 14 & 15 & 9 & 4 & 46 & 20 & 56 & 45 & 18 & 4 & 3 & 3,1 \\
\hline 4 & 26 & 13 & 5 & 2 & 0 & 46 & 130 & 52 & 15 & 4 & 0 & 4 & 4,4 \\
\hline 5 & 3 & 21 & 8 & 9 & 5 & 46 & 15 & 84 & 24 & 18 & 5 & 5 & 3,2 \\
\hline 6 & 4 & 7 & 13 & 19 & 3 & 46 & 4 & 14 & 39 & 76 & 15 & 6 & 3,2 \\
\hline 7 & 1 & 3 & 1 & 23 & 18 & 46 & 1 & 6 & 3 & 92 & 90 & 7 & 4,2 \\
\hline 8 & 9 & 24 & 11 & 2 & 0 & 46 & 45 & 96 & 33 & 4 & 0 & 8 & 3,9 \\
\hline 9 & 12 & 25 & 7 & 0 & 2 & 46 & 60 & 100 & 21 & 0 & 2 & 9 & 4,0 \\
\hline 10 & 6 & 3 & 17 & 18 & 2 & 46 & 6 & 6 & 51 & 72 & 10 & 10 & 3,2 \\
\hline 11 & 4 & 14 & 16 & 10 & 2 & 46 & 20 & 56 & 48 & 20 & 2 & 11 & 3,2 \\
\hline 12 & 1 & 7 & 8 & 21 & 9 & 46 & 1 & 14 & 24 & 84 & 45 & 12 & 3,7 \\
\hline 13 & 14 & 14 & 7 & 8 & 3 & 46 & 14 & 28 & 21 & 32 & 15 & 13 & 2,4 \\
\hline 14 & 7 & 19 & 11 & 9 & 0 & 46 & 35 & 76 & 33 & 18 & 0 & 14 & 3,5 \\
\hline 15 & 4 & 9 & 17 & 10 & 6 & 46 & 4 & 18 & 51 & 40 & 30 & 15 & 3,1 \\
\hline 16 & 6 & 9 & 8 & 19 & 4 & 46 & 30 & 36 & 24 & 38 & 4 & 16 & 2,9 \\
\hline 17 & 2 & 3 & 12 & 16 & 13 & 46 & 2 & 6 & 36 & 64 & 65 & 17 & 3,8 \\
\hline 18 & 23 & 16 & 3 & 4 & 0 & 46 & 115 & 64 & 9 & 8 & 0 & 18 & 4,3 \\
\hline 19 & 4 & 4 & 9 & 21 & 8 & 46 & 4 & 8 & 27 & 84 & 40 & 19 & 3,5 \\
\hline 20 & 3 & 22 & 10 & 10 & 1 & 46 & 15 & 88 & 30 & 20 & 1 & 20 & 3,3 \\
\hline 21 & 7 & 22 & 10 & 4 & 3 & 46 & 35 & 88 & 30 & 8 & 3 & 21 & 3,6 \\
\hline 22 & 2 & 10 & 12 & 19 & 3 & 46 & 2 & 20 & 36 & 76 & 15 & 22 & 3,2 \\
\hline 23 & 0 & 4 & 5 & 14 & 23 & 46 & 0 & 16 & 15 & 28 & 23 & 23 & 1,8 \\
\hline
\end{tabular}


Tabla 3. Promedio de puntuación para cada ítem según la carrera

\begin{tabular}{|c|c|c|c|c|c|c|c|c|c|c|c|c|c|c|c|c|c|c|}
\hline$\stackrel{\Xi}{\varrho}$ & 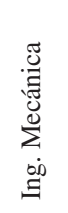 & 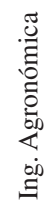 & $\begin{array}{l}\frac{\pi}{00} \\
\frac{0}{0} \\
.0\end{array}$ & 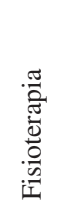 & 晜 & $\begin{array}{l}\stackrel{\pi}{0} \\
:=0 \\
\stackrel{0}{\Sigma}\end{array}$ & $\begin{array}{l}\vec{\Xi} \\
: \\
\text { o. } \\
\Xi\end{array}$ & 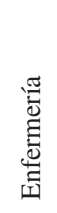 & $\begin{array}{l}\frac{G}{00} \\
0 \\
0 \\
0 \\
0\end{array}$ & 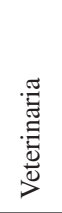 & 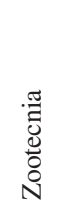 & $\begin{array}{l}\stackrel{\Xi}{0} \\
\stackrel{\Xi}{\Xi} \\
z\end{array}$ & 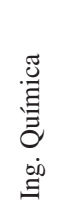 & $\begin{array}{l}\frac{\pi}{60} \\
\frac{0}{0} \\
\frac{0}{0} \\
0 \\
0\end{array}$ & 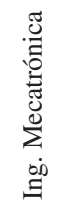 & 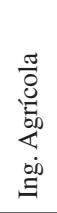 & 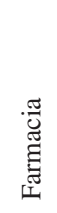 & 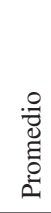 \\
\hline 1 & 2,8 & 3,0 & 3,3 & 2,8 & 4,2 & 3,3 & 2,8 & 2,4 & 3,6 & 2,4 & 2,9 & 3,1 & 4,0 & 2,6 & 3,2 & 3,3 & 3,6 & 3,1 \\
\hline 2 & 3,6 & 3,0 & 3,8 & 3,2 & 4,1 & 3,6 & 3,8 & 3,4 & 3,6 & 3,2 & 3,3 & 3,5 & 3,8 & 3,2 & 3,9 & 2,7 & 3,9 & 3,5 \\
\hline 3 & 2,8 & 3,0 & 2,9 & 3,3 & 4,0 & 2,9 & 2,5 & 2,6 & 2,6 & 2,8 & 2,9 & 3,3 & 3,8 & 2,3 & 2,6 & 3,4 & 3,6 & $\mathbf{3 , 0}$ \\
\hline 4 & 3,7 & 4,3 & 4,3 & 4,3 & 4,9 & 4,5 & 3,9 & 4,2 & 4,5 & 4,6 & 4,5 & 4,5 & 4,7 & 4,1 & 4,2 & 4,3 & 4,7 & 4,4 \\
\hline 5 & 3,3 & 2,8 & 3,1 & 2,5 & 3,7 & 3,3 & 3,4 & 2,8 & 2,9 & 2,9 & 3,0 & 3,2 & 3,8 & 2,9 & 3,5 & 3,1 & 3,6 & 3,2 \\
\hline 6 & 3,0 & 3,0 & 3,0 & 3,4 & 4,0 & 3,6 & 2,9 & 2,6 & 3,5 & 2,7 & 3,1 & 3,1 & 3,9 & 2,6 & 3,1 & 3,2 & 3,6 & 3,2 \\
\hline 7 & 3,6 & 4,2 & 4,0 & 3,8 & 4,7 & 4,1 & 3,6 & 4,0 & 4,2 & 3,8 & 3,9 & 4,1 & 4,7 & 3,5 & 3,5 & 3,9 & 4,3 & 4,0 \\
\hline 8 & 3,6 & 3,7 & 4,2 & 4,3 & 4,6 & 4,0 & 3,8 & 3,6 & 3,9 & 3,8 & 3,7 & 3,9 & 4,4 & 3,5 & 4,2 & 4,1 & 4,2 & 4,0 \\
\hline 9 & 3,7 & 3,8 & 3,9 & 4,3 & 4,5 & 3,9 & 3,9 & 3,8 & 3,8 & 4,0 & 3,9 & 4,3 & 4,6 & 3,4 & 4,1 & 3,9 & 4,2 & 4,0 \\
\hline 10 & 3,2 & 3,1 & 3,5 & 2,6 & 3,7 & 3,5 & 3,1 & 3,0 & 3,6 & 3,3 & 3,2 & 3,3 & 4,0 & 2,8 & 3,0 & 3,1 & 3,8 & 3,3 \\
\hline 11 & 3,3 & 2,9 & 3,2 & 3,6 & 3,9 & 3,7 & 3,4 & 3,3 & 3,4 & 3,2 & 3,2 & 3,7 & 4,1 & 1,9 & 3,4 & 3,2 & 3,6 & 3,4 \\
\hline 12 & 3,8 & 3,2 & 3,9 & 2,8 & 3,9 & 3,8 & 3,9 & 3,4 & 3,6 & 3,7 & 3,6 & 3,3 & 3,7 & 3,5 & 4,0 & 3,3 & 3,7 & 3,6 \\
\hline 13 & 2,8 & 2,2 & 2,8 & 2,0 & 3,2 & 2,4 & 2,7 & 2,3 & 2,5 & 2,1 & 2,6 & 2,1 & 3,2 & 2,7 & 3,1 & 2,2 & 2,6 & 2,6 \\
\hline 14 & 3,3 & 3,2 & 3,6 & 3,3 & 4,0 & 3,5 & 3,5 & 3,1 & 3,2 & 3,1 & 3,5 & 3,4 & 3,8 & 3,3 & 3,5 & 3,4 & 3,8 & 3,4 \\
\hline 15 & 3,5 & 3,3 & 3,4 & 3,5 & 4,8 & 3,3 & 3,2 & 3,3 & 3,1 & 3,2 & 3,2 & 3,8 & 4,5 & 3,1 & 3,0 & 3,5 & 4,0 & 3,5 \\
\hline 16 & 2,8 & 2,9 & 3,1 & 3,3 & 3,3 & 2,7 & 2,6 & 3,2 & 2,9 & 2,9 & 3,3 & 3,3 & 3,4 & 3,2 & 2,8 & 3,2 & 3,5 & $\mathbf{3 , 1}$ \\
\hline 17 & 3,5 & 3,8 & 4,0 & 3,9 & 4,9 & 3,8 & 3,5 & 3,6 & 4,1 & 3,7 & 3,6 & 4,0 & 4,9 & 3,0 & 3,7 & 4,0 & 4,7 & 3,9 \\
\hline 18 & 4,0 & 4,3 & 4,6 & 3,9 & 5,0 & 4,4 & 3,5 & 4,4 & 4,2 & 4,2 & 4,0 & 4,5 & 4,9 & 3,2 & 3,6 & 4,4 & 4,8 & 4,2 \\
\hline 19 & 3,4 & 3,4 & 3,3 & 3,5 & 4,6 & 3,6 & 2,9 & 3,2 & 3,6 & 3,0 & 3,4 & 3,7 & 4,2 & 2,8 & 3,2 & 3,4 & 4,1 & 3,5 \\
\hline 20 & 3,5 & 3,2 & 3,4 & 3,1 & 4,0 & 3,2 & 3,6 & 3,0 & 3,1 & 2,9 & 3,1 & 3,0 & 3,8 & 2,8 & 3,5 & 3,4 & 3,6 & 3,3 \\
\hline 21 & 3,5 & 3,3 & 2,9 & 3,0 & 4,5 & 3,5 & 3,3 & 3,0 & 3,7 & 3,4 & 3,3 & 3,6 & 4,3 & 3,0 & 3,3 & 3,6 & 4,1 & 3,5 \\
\hline 22 & 3,4 & 2,8 & 3,7 & 2,7 & 3,8 & 3,3 & 3,3 & 2,9 & 3,1 & 3,2 & 3,5 & 3,0 & 3,4 & 2,7 & 3,4 & 2,7 & 3,3 & 3,2 \\
\hline 23 & 1,7 & 2,2 & 2,0 & 1,9 & 3,9 & 1,9 & 1,7 & 2,2 & 1,9 & 1,4 & 1,7 & 1,8 & 2,9 & 1,7 & 1,8 & 2,2 & 3,8 & 2,1 \\
\hline
\end{tabular}

de interés y utilidad se comportan mejor en nuestro estudio debido a la población seleccionada, estudiantes involucrados en una carrera universitaria y no estudiantes de final de secundaria que pueden ver la química como una materia más, no como parte de su formación. Con estos resultados tenemos seguridad de que la prueba no presenta errores y puede medir el constructo propuesto.

\section{Actitudes por carreras}

En la Tabla 3 se presentan los resultados obtenidos para cada ítem y para cada carrera. La información globalizada es compleja para ser analizada, por ello debe discutirse de acuerdo a las categorías planteadas.

\section{Actitudes generales en los cursos}

Se tomó el promedio general de todas las carreras para tener una idea global de las actitudes de todos los estudiantes, las cuales están influenciadas por cada carrera de forma particular. En la Figura 2 se observa el promedio de la puntuación dada para cada ítem por toda la población encuestada. Los ítems 4, 7, 8, 9 y 18 proporcionan puntajes que los colocan en el rango de actitudes muy positivas, resaltando sobretodo la importancia de la química en la vida diaria. Al contrastar el ítem 4 (La química sirve para conocer muchos aspectos de nuestra vida cotidian $a$ ) con el ítem 13 (Debo esforzarme mucho para aprender química) se concluye de forma general que, si bien los estudiantes sienten que la química es una ciencia útil, quizá por la divulgación científica de los medios también la consideran una ciencia difícil de aprender, lo cual es corroborado por el ítem 22 (Cuando me enfrento a los problemas de química NO puedo resolverlos con facilidad). Esto proporciona información parcial e inicial para llegar a explicar la razón de apatía y pérdida en los cursos de química, además de considerar ésta como una asignatura externa al programa profesional

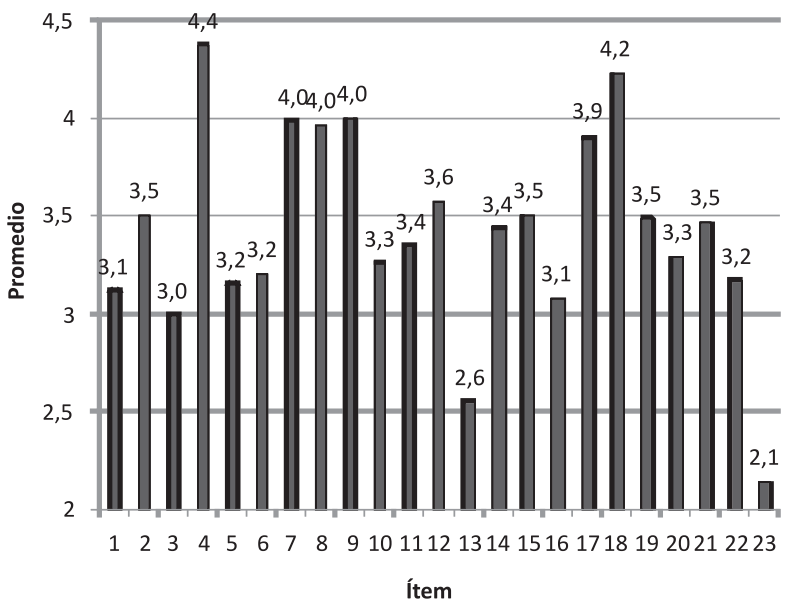

Figura 2. Promedio de las actitudes de la población para cada ítem 
Tabla 4. Valores de las actitudes por categoría para cada carrera

\begin{tabular}{|c|c|c|c|c|c|c|c|c|c|c|c|c|c|c|c|c|c|c|}
\hline $\begin{array}{l}\text { Categoría / } \\
\text { Carrera }\end{array}$ & 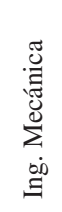 & 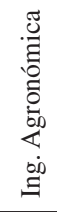 & $\begin{array}{l}\frac{\pi}{60} \\
\frac{0}{0} \\
0 \\
0\end{array}$ & 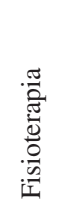 & 总 & 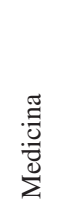 & $\begin{array}{l}\bar{\Xi} \\
\dot{0} \\
\Xi\end{array}$ & 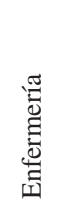 & $\begin{array}{l}\frac{\pi}{60} \\
0 \\
0 \\
0\end{array}$ & & 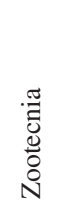 & $\begin{array}{l}\stackrel{0}{0} \\
\stackrel{\Xi}{\Xi} \\
\text { Z }\end{array}$ & 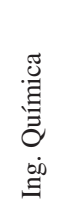 & $\begin{array}{l}\frac{\pi}{60} \\
\frac{0}{0} \\
0 \\
0 \\
0 \\
0\end{array}$ & 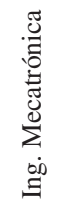 & 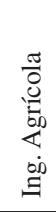 & & 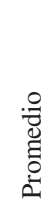 \\
\hline Importancia & 3,4 & 3,5 & 3,7 & 4,0 & 4,3 & 3,8 & 3,5 & 3,6 & 3,7 & 3,7 & 3,7 & 3,9 & 4,2 & 3,2 & 3,7 & 3,7 & 4,0 & 3,8 \\
\hline Dificultad & 3,3 & 2,9 & 3,4 & 2,8 & 3,8 & 3,2 & 3,4 & 2,9 & 3,1 & 2,9 & 3,2 & 3,0 & 3,6 & 2,9 & 3,5 & 2,9 & 3,4 & 3,2 \\
\hline Interés & 3,3 & 3,3 & 3,4 & 3,3 & 4,4 & 3,5 & 3,2 & 3,1 & 3,6 & 3,2 & 3,3 & 3,6 & 4,2 & 2,9 & 3,3 & 3,5 & 3,9 & 3,5 \\
\hline Utilidad & 2,9 & 3,2 & 3,3 & 2,8 & 4,2 & 3,3 & 2,7 & 3,2 & 3,3 & 2,9 & 2,9 & 3,2 & 3,9 & 2,6 & 2,8 & 3,2 & 4,1 & 3,2 \\
\hline
\end{tabular}

de muchas carreras (ítem 16: Todas las carreras de la universidad deberían tener al menos un curso de química).

Sólo 6 ítems pueden clasificarse dentro de las actitudes muy positivas $(4,7,8,9,17$ y 18), mostrando puntuaciones muy importantes. Vale la pena resaltar los resultados de los ítems 8 y 9, ya que la química suele asociarse comunmente a peligros para el medio ambiente, a contaminación y a riesgos para la salud; pero en este caso los estudiantes le confieren un efecto positivo y la asocian con posibilidad de superación. Sin embargo, la mayoria de estudiantes aprecian el curso de química y lo consideran útil, aunque tambien difícil.

El ítem 23 corresponde al de menor valor $(2,1)$, lo cual no es extraño teniendo en cuenta que la población corresponde a estudiantes que ya han elegido una carrera, y por tanto el cambio de carrera es un evento poco probable dentro del concurso de las aspiraciones y proyecciones de los estudiantes encuestados.

\section{Resultados por carrera}

En la Tabla 4 y Figura 3 aparecen los valores promedio por categorías para cada carrera, los cuales se obtienen a partir de la Tabla 3. La carrera de Química posee los valores más altos, lo cual nos sirve como referente para cualquier comparación, y luego le siguen Ingeniería Química y Farmacia (química farmacéutica). Las categorías para Química estan en el orden de "muy positivas", excepto para la "dificultad", la cual corresponde en la mayoría de carreras al valor más bajo, al lado de la "utilidad". La dificultad posee un promedio general de 3,2 (moderadamente positivo, cercano al valor neutral). Esta categoría posee las preguntas 2, 5, 13, 14,20 y 22. Los ítems 2 y 14 son muy similares y tienen que ver con un problema que afronta la enseñanza de la química, sobre todo a nivel de principiantes: el lenguaje que se utiliza. El lenguaje de la química posee una dificultad relacionada con su objeto de estudio y las representciones que las

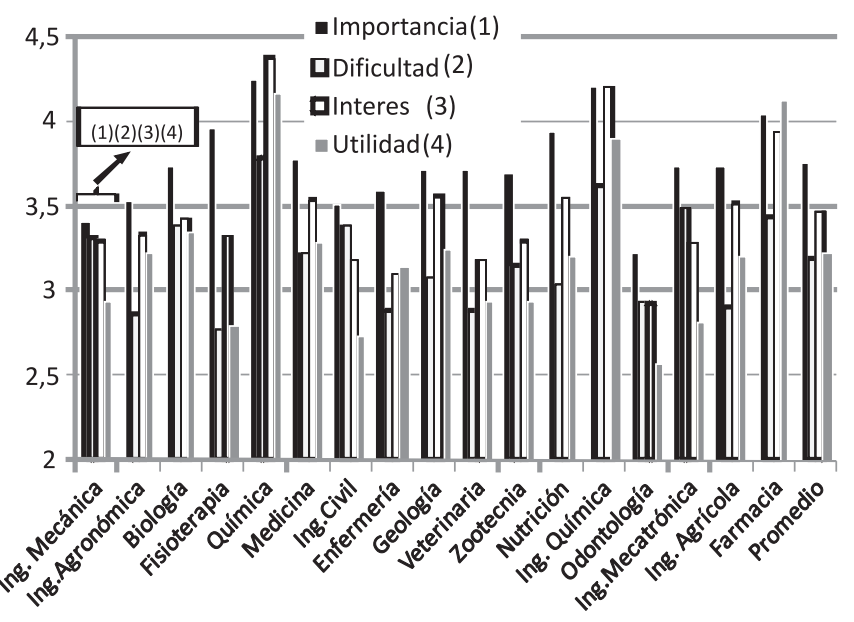

Figura 3. Valores de actitudes por categoría para cada carrera personas hacen de éste. De hecho, para describir los cambios de la materia debemos acudir a tres aspectos: macroscópico, microscópico y simbólico. La estrecha relación entre estos tres aspectos representa una dificultad para un estudiante en la medida en que lo único visible es lo macroscópico, lo demás debe imaginarlo e incorporarlo estableciendo las relaciones respectivas, todo en conjunto mediado por el lenguaje. ${ }^{17}$

De la misma forma, los ítems 5 y 22 son similares y por ello para la población estudiada obtienen el mismo promedio $(3,2)$. Las carreras de Ingeniería Agronómica, Ingeniería Agrícola, Enfermería, Fisioterapia y Odontología marcan, en ambas preguntas, actitudes negativas (Tabla 3), igual a lo ocurrido con la categoría "dificultad" en general (Tabla 4), y similar a lo que encuentran Salta y Tzougraki ${ }^{9}$ en estudiantes de secundaria. En suma, los resultados indican que la química para los estudiantes no es fácil pero tampoco representa un reto imposible, la colocan en un punto donde la pueden sobrellevar, donde el interés puede marcar la diferencia y permitirles sacar adelante el curso: realizando todos los esfuerzos posibles para enfrentar la dificultad e impidiendo que ésta los domine.

Finalizando la categoría de dificultad, se observa que el bajo valor asignado está muy influenciado por el valor obtenido para el ítem 13 (2,6) (Debo esforzarme mucho para aprender química), en contraste con el valor del ítem 20 (3,3) (Comprendo los conceptos de química con facilidad). Estas tendencias son entendibles cuando se correlaciona la labor docente con lo manifestado por los estudiantes: las clases de química y la comprensión de los conceptos no son difíciles; es decir luego de una clase los estudiantes suelen adquirir la idea de que han comprendido las explicaciones, pero la dificultad radica en relacionar todo el conjunto de conceptos y habilidades sugeridas, en enfrentar con fortaleza cognitiva un problema de química y aplicar todos los conceptos, o sea en establecer y aplicar una red coherente de conceptos. En consecuencia, el desarrollo de las clases de química requiere de la aplicación de ciertas actividades integradoras que fortalezcan las habilidades y la red conceptual de los estudiantes, más allá del aprendizaje sencillo y aislado de conceptos y principios. Continuando con otra categoría, para casi todas las carreras la "importancia" de la química es lo más relevante; como se vio anteriormente, consideran que la química posee una característica especial para resolver problemas reales con beneficios sociales (ítem 8), de forma similar a lo hallado por Salta y Tzougraki, donde los estudiantes resaltan que la química resuelve problemas ambientales y mejora nuestras vidas. ${ }^{9}$ Lo anterior puede ser el resultado de una relación directa entre enseñanza e importancia, pues comunmente se desarrollan clases donde resaltamos las cuestiones que la química ha afrontado y resuelto para bien común (síntesis de fertilizantes, obtención de vacunas, análisis de venenos, elaboración de polímeros, etc).

De otro lado, la carrera de Odontología le confiere la menor importancia a la química (Figura 3), aunque sus egresados utilizan moderadamente esta ciencia en su profesión (productos como resinas, materiales dentales, blanqueadores, cremas, procedimientos prepa- 
rativos, etc). En general, para las carreras de la salud (Fisioterapia, Medicina, Odontología, Enfermeria y Nutrición) todas las categorías presentan valores bajos, pero son quienes le confieren valores más bajos a la dificultad y a la importancia del curso de química.

Con respecto al "interés", el ítem 1 (El curso de química me parece más agradable que otros cursos) aparece como un importante indicador respecto al agrado que sienten por estar en el curso. Las carreras de Química (puntaje de 4,2), Geología (3,6), Ingeniería Química $(4,0)$ y Farmacia $(3,6)$ poseen una actitud claramente positiva hacia el curso, las demás carreras permanecen neutrales ante el curso, no poseen una actitud positiva pero tampoco negativa, dejando en duda sus ideas al respecto. Con el ítem 3 (Me gustaría tener clases de química con mayor frecuencia) muestran negativamente que no desean esta posibilidad, y aunque reconocen que el conocimiento en química es útil en algún momento de sus carreras (ítem 7) quizás prefieren esperar, no por incapacidad de ellos (ítem 12, La química es una ciencia muy compleja para mi nivel de conocimientos, con 3,6 ) sino por la dificultad que han manifestado. La puntuación en el ítem 17 (Detesto los cursos de química, 3,9) da un alivio al saber que estos estudiantes no poseen sentimientos de odio o rechazo hacia el curso, representado de alguna forma por la imagen de su profesor y los contenidos desarrollados.

Finalmente, sobre la "utilidad" de la química el valor promedio de 3,2 (Tabla 4) la coloca casi en un valor neutral, cercano a las actitudes negativas. El valor obtenido se encuentra afectado por el ítem 23, como se mencionó antes. Observando los valores de la Tabla 4, las carreras de Química, Farmacia e Ingeniería Química muestran actitudes muy positivas, algo importante para personas que se van a dedicar a la química, y el resto de carreras muestran que están en lo suyo, descartando a la química como parte de sus estudios. Sobre los ítems de utilidad, 10, 18 y 23, el 10 (La seguridad de mi futuro es independiente del conocimiento químico) con valor promedio de 3,3 indica que para las profesiones en general no se considera estrictamente necesario el saber en química para alcanzar un desempeño profesional aceptable, mientras que con el ítem 18 (Los conocimientos en química son necesarios en el desarrollo de mi carrera) el valor de 4,2 muestra que los estudiantes son muy conscientes que la química está implicada en su carrera. Para la carrera de Química los dos ítems marcan 3,7 y 5, interpretándose que para los estudiantes no basta con saber un conocimiento para desarrollarse en un campo aún cuando esos conocimientos sean muy necesarios para sacar una profesión adelante.

\section{CONCLUSIONES}

Desde los resultados obtenidos, la población estudiada considera útil la química, aunque no para involucrarse en su estudio, sino para comprender la naturaleza. La utilidad no transciende al interés por estudiarla, ya que la dificultad es vista como un obstáculo actitudinal al enfrentarse a la química, lo cual se convierte en un llamado urgente a profesores para reflexionar sobre la necesidad de revisar las metodologías de enseñanza y evaluación, ya que si bien aprender requiere esfuerzo, la dificultad indicada aquí va más allá de la falta de interés, pues aun los estudiantes de mejor actitud la consideran como algo negativo (carrera de química). Es necesario atender las diversas necesidades de las profesiones, teniendo en cuenta que, lo que a un químico le parece interesante le puede resultar sin importancia a otro profesional (por ejemplo, tratar el equilibrio químico desde una perspectiva extremadamente cuantitativa, puede ser útil para el químico, pero al profesional de la salud le interesará posiblemente más el aspecto cualitativo, en sistemas como el metabolismo, respiración o capacidad amortiguadora de la sangre).

La actitud hacia la química de la población estudiada no enciende alarmas pero nos deja en una posición de confort la cual si no es atendida puede pasar a una posición negativa de actitudes. En general existen actitudes positivas, pero debemos mejorar las percepciones sobre la dificultad y la utilidad que se le confieren a la química. Nuestros estudiantes pueden poseer mejores desempeños si atendemos esas categorías actitudinales, realizando propuestas que tengan en cuenta la mayor parte de los intereses involucrados.

Para cerrar este trabajo invitamos a los profesores involucrados en la enseñanza de la química para que apliquen la encuesta, ya validada, y tengan siempre en cuenta el componente actitudinal en el desarrollo de sus cursos de química.

\section{AGRADECIMIENTOS}

A la Universidad Nacional de Colombia, y a todos los profesores que participaron en las diferentes actividades del trabajo y la aplicación de encuestas.

\section{REFERENCIAS}

1. Cheung, D.; Res. Sci. Educ. 2009, 39, 75.

2. Cukrowska, E.; Staskun, M. G.; Schoeman, H. S.; South African J. Chem. 1999, 52, 8 .

3. Wang, T. L.; Berlin, D.; Int. J. Sci. Educ. 2010, 32, 2413.

4. Osborne, J. F.; Simon, S.; Collins, S.; Int. J. Sci. Educ. 2003, 25, 1049.

5. Marbà-Tallada, A.; Márquez-Bargalló, C.; Enseñanza de las ciencias 2010, 28, 19.

6. Molina, M.; Farias, D.; Carriazo, J.; Enseñanza de las ciencias 2009, Número Extra VIII, 904

7. Kind, P. M.; Jones, K.; Barmby, P.; Int. J. Sci. Educ. 2007, 29, 871.

8. Shannon, A. G.; Sleet, R. J.; Stern, W.; Aust. Sci. Teachers J. 1982, 28, 77.

9. Salta, K.; Tzougraki, C.; Sci. Educ. 2004, 88, 535.

10. Barnes, G.; McInerney, D. M.; Marsh, H. S.; Aust. Educ. Res. 2005, 32, 1.

11. Vázquez, A.; Manassero, M. A.; Rev. Eureka Enseñ. Divul. Cien. 2008, $5,274$.

12. Ajzen, I.; Annu. Rev. Psychol. 2001, 52, 27.

13. Reid, N.; Res. Sci. Technol. Educ. 2006, 24, 3.

14. Hambleton, R. K. In Methodological advances in large scale crossnational educational surveys; Porter, A.; Gamoran, A., eds.; National Academy of Sciences: Washington, 2002, p. 58.

15. Barmby, P.; Kind, P.; Jones, K.; Int. J. Sci. Educ. 2008, 30, 1075.

16. Vázquez, A.; Manassero, M. A.; Enseñanza de las ciencias 1997, 15, 199.

17. Johnstone, A. H.; J. Comput. Assist. Lear. 1991, 7, 75. 